in vivo $35: 3423-3430(2021)$

doi:10.21873/invivo.12642

\title{
Significance of Glasgow Prognostic Scores in NSCLC Patients Treated With Immunotherapy After Platinum-based Cytotoxic Chemotherapy
}

\author{
HYE SEON KANG ${ }^{1}$, AH YOUNG SHIN ${ }^{2}$, CHANG DONG YEO ${ }^{3}$, SUNG KYOUNG KIM ${ }^{4}$, \\ CHAN KWON PARK ${ }^{5}$, JU SANG KIM ${ }^{2}$, SEUNG JOON KIM ${ }^{6}$, SANG HAAK LEE ${ }^{3}$ and JIN WOO KIM ${ }^{7}$ \\ ${ }^{I}$ Division of Pulmonary, Allergy and Critical Care Medicine, Department of Internal Medicine, \\ Bucheon St. Mary's Hospital, College of Medicine, The Catholic University of Korea, Seoul, Republic of Korea; \\ ${ }^{2}$ Division of Pulmonary and Critical Care Medicine, Department of Internal Medicine, \\ Incheon St. Mary's Hospital, College of Medicine, The Catholic University of Korea, Seoul, Republic of Korea; \\ ${ }^{3}$ Division of Pulmonary, Critical Care and Sleep Medicine, Department of Internal Medicine, \\ Eunpyeong St. Mary's Hospital, College of Medicine, The Catholic University of Korea, Seoul, Republic of Korea; \\ ${ }^{4}$ Division of Pulmonary and Critical Care Medicine, Department of Internal Medicine, \\ St. Vincent's Hospital, College of Medicine, The Catholic University of Korea, Seoul, Republic of Korea; \\ ${ }^{5}$ Division of Pulmonary and Critical Care Medicine, Department of Internal Medicine, Yeouido St. Mary's Hospital, \\ College of Medicine, The Catholic University of Korea, Seoul, Republic of Korea; \\ ${ }^{6}$ Division of Pulmonary, Allergy and Critical Care Medicine, Department of Internal Medicine, \\ Seoul St. Mary's Hospital, College of Medicine, The Catholic University of Korea, Seoul, Republic of Korea; \\ ${ }^{7}$ Division of Pulmonary, Critical Care and Sleep Medicine, Department of Internal Medicine, \\ Uijeongbu St. Mary's Hospital, College of Medicine, The Catholic University of Korea, Seoul, Republic of Korea
}

\begin{abstract}
Background/Aim: Little is known about the prognostic role of the Glasgow prognostic score (GPS) in nonsmall cell lung cancer (NSCLC) patients treated with immunotherapy after platinum-based cytotoxic chemotherapy. Patients and Methods: This study used a lung cancer cohort of the Catholic Medical Center of Korea between January 2018 and September 2020. Results: A total of 78 patients with NSCLC treated with immunotherapy as second or further-line therapy were included. Higher GPS values were significant predictors of shorter immune-related progression-free survival (irPFS) and overall survival (OS). The hazard ratios for irPFS were 0.249 for programmed death-ligand 1 (PD-L1) expression $\geq 50 \%$ and 9.73 for a GPS of 2. Older age, lower
\end{abstract}

This article is freely accessible online.

Correspondence to: Dr. Jin Woo Kim, Division of Pulmonary, Critical Care and Sleep Medicine, Department of Internal Medicine, Uijeongbu St. Mary's Hospital, College of Medicine, The Catholic University of Korea, 271, Cheonbo-Ro, Uijeongbu-si, Gyeonggido, 11765, Republic of Korea. Tel: +82 318203995, Fax: +82 318472719, e-mail: medkjw@ catholic.ac.kr

Key Words: Immunotherapy, albumin, C-reactive protein, non-small cell lung cancer.
PD-L1 expression and higher GPS values were independently associated with shorter OS. Conclusion: Higher GPS values were identified as a poor prognostic factor for OS and irPFS in NSCLC patients who received immunotherapy as second or further-line therapy.

The introduction of immune-checkpoint inhibitors (ICIs) into the therapy for non-small cell lung cancer (NSCLC) has transformed the therapeutic landscape of metastatic NSCLC (1). The expression of programmed death-ligand 1 (PD-L1) on tumor cells and tumor mutation burden (TMB) have been used to select patients suitable for ICIs but not all patients with these predictive factors benefit from ICIs (2). However, the proper predictive factors for using ICIs to treat NSCLC patients have not been developed.

Inflammation is an important factor in tumor progression and is associated with poor response to treatment. The response to cancer treatment depends not only on the tumor's characteristics and tumor stage but also on patient-related factors including nutrition and inflammation status (3). Recently, systemic inflammatory response to predict progression and survival has been reported in patients with malignancies. Thus, cancer-related prognosis has been validated using inflammatory markers such as the neutrophillymphocyte ratio (NLR) or the lung immune prognostic index 


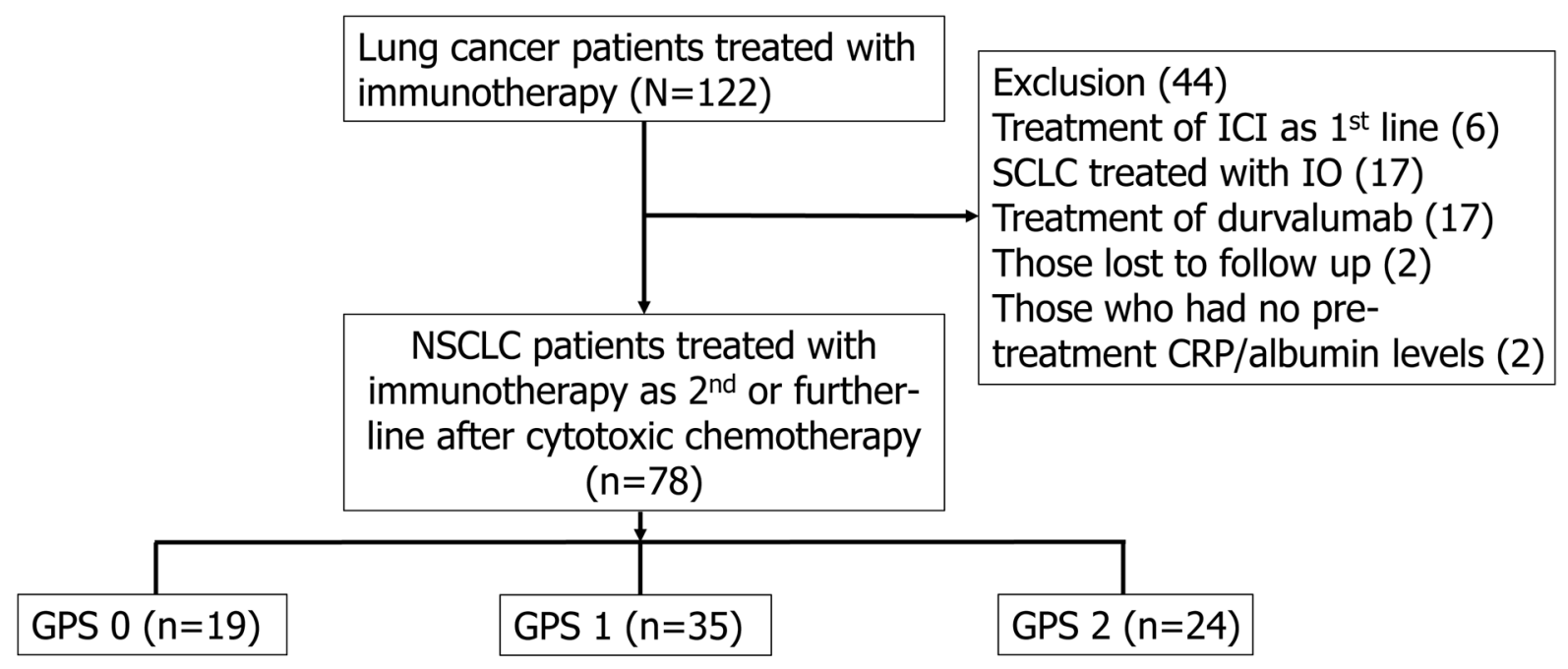

Figure 1. Study flow diagram. ICI, Immune-checkpoint inhibitors; SCLC, small cell lung cancer; CRP, C-reactive protein; NSCLC, non-small cell lung cancer; GPS, Glasgow prognostic score.

consisting of the NLR and lactate dehydrogenase (LDH) levels, and the systemic inflammation response index $(2,4)$.

The Glasgow prognostic score (GPS), reflecting the host systemic inflammatory response and immune status has been validated as a prognostic factor in many malignancies. The GPS is the combination of the C-reactive protein (CRP) concentration (>10 mg/l) and hypoalbuminemia ( $<35 \mathrm{~g} / \mathrm{l})(5$, $6)$. The association of the GPS and ICIs in lung cancer patients has been explored previously; however, previous studies have had small sample sizes or evaluated the posttreatment GPS in lung cancer patients treated with ICIs.

Few studies have evaluated the prognostic value of the GPS in metastatic NSCLC patients treated with ICIs who received at least one regimen of cytotoxic chemotherapy before the administration of ICIs $(7,8)$. Recently Takamori et al. reported the clinical utility of pre-treatment GPS in advanced or recurrent NSCLC patients treated with ICIs but included patients treated with ICIs as first line treatment.(9) In this study, we retrospectively analyzed the survival and immune-related progression-free survival (irPFS) data of patients with metastatic NSCLC and explored the prognostic role of the GPS in these patients.

\section{Patients and Methods}

Patients. We used the lung cancer registry database of the Catholic Medical Center, Seoul, Republic of Korea. Since October 2014, seven hospitals of the Catholic University of Korea (Seoul St. Mary's Hospital, Yeouido St. Mary's Hospital, Eunpyeong St. Mary's Hospital, Uijeongbu St. Mary's Hospital, Bucheon St. Mary's Hospital, Incheon St. Mary's Hospital and St. Vincent's Hospital) have consecutively enrolled lung cancer patients. Clinical information including stage, pathology, treatment modality and survival was systematically recorded by qualified managers to improve data accuracy. The researchers were permitted access by newly assigned serial numbers and anonymizing the dataset.

Patients were eligible for the study if they were diagnosed from January 2018 to March 2020 with histologically confirmed NSCLC and received ICIs as second-line or further-line therapy after treatment failure with platinum-based cytotoxic chemotherapy. Patients who received ICIs as first-line therapy, were diagnosed with small cell lung cancer, received post-chemoradiation consolidation treatment with durvalumab, were lost to follow up, or had no pre-treatment CRP and albumin levels were excluded. The study flow is summarized in Figure 1. The follow-up period ended on September 30, 2020.

This study was approved by the Clinical Research Ethics Committee of the Catholic Medical Center (approval number: XC20RIDI0192). All methods were performed in accordance with the Declaration of Helsinki, participants were informed about the study, the handling of personal data and how confidentiality would be maintained in the management of material and in publishing and presenting results. Researchers were permitted to conduct this study by accessing dataset newly assigned with a serial number whose personal information was removed after ethical approval. We included only patients over 19 years old and written informed consent was obtained from all patients prior to registry enrollment.

Data. We extracted the following data from the patient medical records: patient demographics, smoking history, stage of lung cancer, Eastern Cooperative Oncology Group performance status, laboratory data, history of chemotherapy and/or radiation, survival status, and the dates of disease progression and death. Blood samples drawn within one week prior to ICI treatment were used to compile a pretreatment GPS for each patient using the laboratory values. The patients were classified into three groups based on GPS values as follows: (I) GPS of 2 , elevated CRP level $(>1.0 \mathrm{mg} / \mathrm{dl})$ and hypoalbuminemia $(<3.5$ $\mathrm{g} / \mathrm{dl}$ ); (II) GPS of 1, elevated CRP level or hypoalbuminemia; and (III) 
Kang et al: GPS and Immunotherapy in NSCLC

Table I. Baseline characteristics of NSCLC patients treated with immunotherapy according to GPS values.

\begin{tabular}{|c|c|c|c|c|}
\hline Variables & $\mathrm{GPS}=0 \quad(\mathrm{n}=19)$ & GPS $=1 \quad(n=35)$ & GPS $=2(n=24)$ & $p$-Value \\
\hline Age & $66.74 \pm 9.26$ & $66.77 \pm 9.04$ & $66.88 \pm 9.77$ & 0.999 \\
\hline Gender, male & $14(73.7)$ & $28(80.0)$ & $22(91.7)$ & 0.285 \\
\hline Smoking history & & & & 0.753 \\
\hline Never-smoker & $3(15.8)$ & $6(31.6)$ & $10(52.6)$ & \\
\hline Ever-smoker & $3(8.6)$ & $14(40.0)$ & $18(51.4)$ & \\
\hline Current-smoker & $1(4.2)$ & $10(41.7)$ & $13(54.2)$ & \\
\hline Pack-years & $49.75 \pm 24.59$ & $36.29 \pm 19.73$ & $40.72 \pm 17.31$ & 0.102 \\
\hline Performance status $\geq 2$ & $10(41.7)$ & $11(45.8)$ & $2(8.3)$ & 0.300 \\
\hline Histologic type & & & & 0.773 \\
\hline Adenocarcinoma & $8(42.1)$ & $18(51.4)$ & $14(58.3)$ & \\
\hline Squamous cell carcinoma & $10(52.6)$ & $15(42.9)$ & $10(41.7)$ & \\
\hline Others & $1(5.3)$ & $2(5.7)$ & $0(0.0)$ & \\
\hline Treatment line & & & & 0.121 \\
\hline Second & $15(78.9)$ & $32(91.4)$ & $20(83.3)$ & \\
\hline$\geq$ Third & $4(21.0)$ & $3(8.6)$ & $4(16.7)$ & \\
\hline ICIs & & & & 0.042 \\
\hline Pembrolizumab & $9(47.4)$ & $20(57.1)$ & $11(45.8)$ & \\
\hline Nivolumab & $1(5.3)$ & $6(17.1)$ & $9(37.5)$ & \\
\hline Atezolizumab & $9(47.4)$ & $9(25.7)$ & $4(16.7)$ & \\
\hline EGFR mutation, positive & $0(0.0)$ & $2(5.9)$ & $1(4.2)$ & 0.791 \\
\hline ALK rearrangement, positive & $0(0.0)$ & $0(0.0)$ & $0(0.0)$ & \\
\hline PD-L1 expression & & & & 0.508 \\
\hline $0 \%$ & $4(21.1)$ & $3(8.6)$ & $3(12.5)$ & \\
\hline $1-10 \%$ & $4(21.1)$ & $8(22.8)$ & $4(16.7)$ & \\
\hline $11-49 \%$ & $3(15.8)$ & $3(8.6)$ & $5(20.8)$ & \\
\hline$\geq 50 \%$ & $6(31.6)$ & $21(60.0)$ & $12(50.0)$ & \\
\hline
\end{tabular}

Data are presented as mean \pm standard deviation (range) or number (\%). GPS, Glasgow prognostic score; NSCLC, non-small cell lung cancer; ICI, immune check-point inhibitor; EGFR, epidermal growth factor receptor; ALK, anaplastic lymphoma receptor tyrosine kinase; PD-L1, programmed death-ligand 1.

GPS of 0 , neither elevated CRP level nor hypoalbuminemia $(5,10)$. The serum CRP levels were measured using immunoturbidimetric assays (CRPL3, Roche Diagnostics, Indianapolis, IN, USA).

Statistical analysis. The patient baseline demographics and clinical outcomes were compared according to the GPS. We used Pearson's chi-squared test to compare the discrete variables and the Student's $t$-test or analysis of variance to compare the continuous variables. Kaplan-Meier survival analysis was used to compare OS and irPFS according to GPS. Differences in survival and irPFS were determined by the log-rank test. The Mann-Whitney test was used to compare the median values. Hazard ratios (HRs) and the corresponding 95\% confidence intervals (CIs) were calculated for the predictors that were significant in multivariate Cox regression analysis. A two-sided $p$-value of $<0.05$ was considered statistically significant. All statistical analyses were performed using SPSS for Windows software (ver. 20.0; IBM Corp., Armonk, NY, USA) (11).

\section{Results}

Patient characteristics. Overall, 122 NSCLC patients treated with ICIs were enrolled, of whom 44 met the exclusion criteria (treatment with ICIs as first-line therapy, small cell lung cancer treated with ICIs, treatment with durvalumab, and patients lost to follow-up, or those who had no pretreatment CRP/albumin levels). Thus, 78 patients were finally included in our analysis (Figure 1). The mean age of the included subjects was 67.1 \pm 9.17 years (range $=38.0-84.0$ years). There were $64(82.1 \%)$ males. Of all the included patients, $19(24.4 \%), 35(44.9 \%)$, and 24 (30.8\%) were classified into the GPS 0, GPS 1 , and GPS 2 groups, respectively. We compared these three groups and explored the clinical factors predicting treatment outcomes including OS and irPFS. The total and median follow-up times were $12.7,14.2,15.0$, and 6.4 person-months, respectively.

The baseline characteristics of these three groups are summarized in Table I. The mean age and smoking history were not different between the three groups. The proportion of poor performance status, histologic type, treatment line of ICIs, driving mutations, and PD-L1 expression were similar between the three groups. However, the GPS 0 group tended to receive atezolizumab and the GPS 2 group tended to receive pembrolizumab as ICIs $(p=0.042)$.

Prognostic analysis. The GPS 2 group displayed a shorter median irPFS than the GPS 0 or GPS 1 group [23.0 (95\% 
A

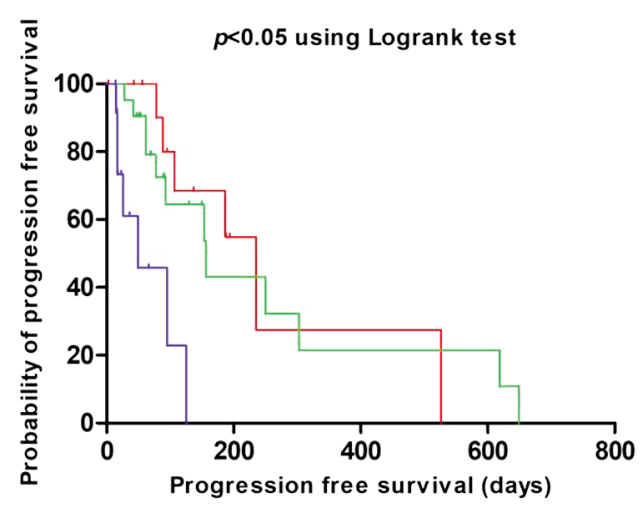

B

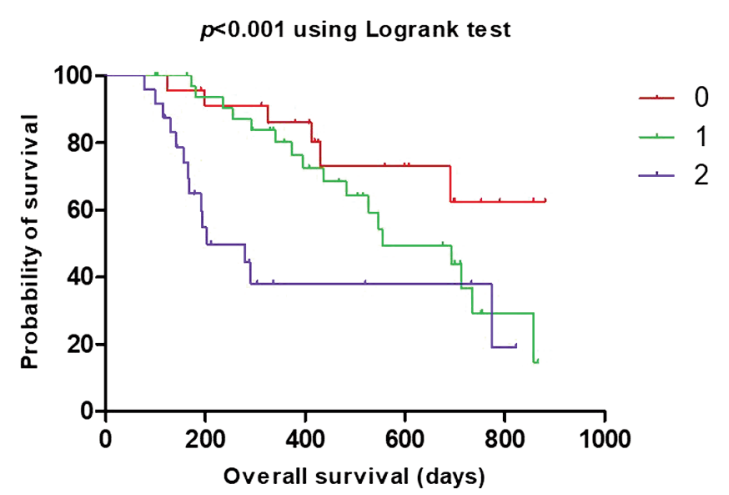

Figure 2. Clinical outcomes according to Glasgow prognostic score (GPS) in non-small cell lung cancer (NSCLC) patients treated with immunotherapy after platinum-based cytotoxic chemotherapy.

Table II. Univariate and multivariate analysis for predicting progression-free survival in NSCLC patients treated with immunotherapy after platinumbased cytotoxic chemotherapy.

\begin{tabular}{|c|c|c|c|c|c|c|}
\hline \multirow[b]{2}{*}{ Variables } & \multicolumn{3}{|c|}{ Univariate analysis } & \multicolumn{3}{|c|}{ Multivariate analysis } \\
\hline & HR & $95 \% \mathrm{CI}$ & $p$-Value & HR & $95 \% \mathrm{CI}$ & $p$-Value \\
\hline Age & 0.988 & $0.956-1.021$ & 0.480 & & & \\
\hline Gender, male & 1.637 & $0.802-3.341$ & 0.176 & & & \\
\hline Smoking status & & & 0.255 & & & \\
\hline Ex-smoker & 2.204 & $0.812-5.985$ & 0.121 & & & \\
\hline Current smoker & 1.578 & $0.586-4.248$ & 0.367 & & & \\
\hline Pack-years & 1.004 & $0.991-1.017$ & 0.550 & & & \\
\hline Stage & 1.774 & $0.973-3.235$ & 0.061 & 1.083 & $0.530-2.211$ & 0.827 \\
\hline \multicolumn{7}{|l|}{ Histological cell type } \\
\hline \multicolumn{7}{|l|}{ Adenocarcinoma } \\
\hline Squamous cell carcinoma & 1.261 & $0.293-5.422$ & 0.755 & & & \\
\hline Adenosquamous cell carcinoma & 0.82 & $0.189-3.553$ & 0.791 & & & \\
\hline NOS & 0.403 & $0.036-4.534$ & 0.462 & & & \\
\hline PD-L1 & & & 0.014 & & & 0.015 \\
\hline \multicolumn{7}{|l|}{$0 \%$} \\
\hline $1-10 \%$ & 1.082 & $0.369-3.169$ & 0.886 & 0.38 & $0.109-1.329$ & 0.130 \\
\hline $11-49 \%$ & 1.619 & $0.569-4.608$ & 0.367 & 1.124 & $0.357-3.545$ & 0.842 \\
\hline$\geq 50 \%$ & 0.436 & $0.170-1.119$ & 0.084 & 0.249 & $0.084-0.739$ & 0.012 \\
\hline GPS & & & $<0.001$ & & & $<0.001$ \\
\hline \multicolumn{7}{|l|}{0} \\
\hline 1 & 1.051 & $0.479-2.305$ & 0.902 & 2.146 & $0.814-5.656$ & 0.123 \\
\hline 2 & 5.011 & $2061-12.187$ & $<0.001$ & 973 & $2931-32.298$ & $<0.001$ \\
\hline
\end{tabular}

NSCLC, Non-small cell lung cancer; HR, hazard ratio; CI, confidence interval; NOS, not otherwise specified; PD-L1, programmed death-ligand 1; GPS, Glasgow prognostic score.

$\mathrm{CI}=12.43-33.57)$ days $v s .89 .0(95 \% \mathrm{CI}=57.6-120.4)$ days $v$. 107.0 (95\% CI=41.92-172.1) days respectively, $p<0.001]$. Also, the GPS 2 group showed significantly shorter median OS than the GPS 0 or GPS 1 group [412.9 (95\% CI=278.5547.27) days vs. 593.7 (95\% CI=504.3-683.2) days in GPS
0 and 768.5 (95\% CI=655.7-881.2) days in GPS $1, p<0.001]$ (Figure 2).

In the multivariate analysis of irPFS, the HR was 0.249 (95\% CI $=0.084-0.739, p=0.012$ ) for PD-L1 expression $\geq 50 \%$ and $9.73(95 \% \mathrm{CI}=2.931-32.298, p<0.001)$ for GPS 2 (Table 
Table III. Univariate and multivariate analysis for predicting overall survival in NSCLC patients treated with immunotherapy after platinum-based cytotoxic chemotherapy.

\begin{tabular}{|c|c|c|c|c|c|c|}
\hline \multirow[b]{2}{*}{ Variable } & \multicolumn{3}{|c|}{ Univariate analysis } & \multicolumn{3}{|c|}{ Multivariate analysis } \\
\hline & HR & $95 \% \mathrm{CI}$ & $p$-Value & HR & $95 \% \mathrm{CI}$ & $p$-Value \\
\hline Age & 1.060 & $1.016-1.107$ & 0.007 & 1.067 & $1.005-1.133$ & 0.033 \\
\hline Sex, male & 2.652 & $0.934-7.531$ & 0.067 & 1.305 & $0.282-6.032$ & 0.733 \\
\hline Smoking status & & & 0.175 & & & \\
\hline Ex-smoker & 3.633 & $0.842-15.683$ & 0.084 & & & \\
\hline Current smoker & 2.530 & $0.575-11.133$ & 0.220 & & & \\
\hline Pack-years & 1.014 & 0.998-1.029 & 0.085 & 1.015 & $0.998-1.033$ & 0.083 \\
\hline Stage & 1.492 & $0.757-2.942$ & 0.248 & & & \\
\hline $\begin{array}{l}\text { Histologic cell type } \\
\text { Adenocarcinoma }\end{array}$ & & & 0.388 & & & \\
\hline $\begin{array}{l}\text { Adenocarcinoma } \\
\text { Squamous cell carcinoma }\end{array}$ & 1.005 & $0.515-1.959$ & 0.989 & & & \\
\hline $\begin{array}{l}\text { Adenosquamous cell carcinoma } \\
\text { NOS }\end{array}$ & 3.618 & $0.822-15.919$ & 0.089 & & & \\
\hline $\begin{array}{l}\text { PD-L1 } \\
0 \%\end{array}$ & & & 0.007 & & & 0.036 \\
\hline $1-10 \%$ & 0.550 & $0.145-2.081$ & 0.379 & 0.263 & $0.053-1.294$ & 0.100 \\
\hline $11-49 \%$ & 3.290 & $0.957-11.309$ & 0.059 & 1.455 & $0.361-5.874$ & 0.598 \\
\hline$\geq 50 \%$ & 0.776 & $0.261-2.309$ & 0.649 & 0.364 & $0.094-1.410$ & 0.144 \\
\hline $\begin{array}{c}\text { GPS } \\
0\end{array}$ & & & 0.002 & & & 0.007 \\
\hline 1 & 3.513 & $1.028-12.007$ & 0.045 & 4.498 & $1.164-17.388$ & 0.029 \\
\hline 2 & 8.459 & $2.402-29.793$ & 0.001 & 8.574 & $2.218-33.140$ & 0.002 \\
\hline
\end{tabular}

NSCLC, Non-small cell lung cancer; HR, hazard ratio; CI, confidence interval; NOS, not otherwise specified; PD-L1, programmed death-ligand 1; GPS, Glasgow prognostic score.

II). In multivariate analysis for OS, the HR was 0.189 for PD-L1 expression between 1 and 10\%, 0.297 for PD-L1 expression $\geq 50 \%, 4.247$ in the GPS 1 group, and 9.710 in the GPS 2 group (Table III).

\section{Discussion}

In the present study, we compared the clinical outcomes of NSCLC patients treated with ICIs as second-line or further-line therapy after treatment failure with platinum-based cytotoxic chemotherapy according to the GPS values and explored the prognostic role of GPS values for treatment outcomes. We found that the baseline characteristics were not different according to GPS. Patients with higher GPS values displayed shorter median irPFS and OS than those in the GPS 0 or GPS 1 groups. In multivariate analysis, lower PD-L1 expression and higher GPS was associated with shorter irPFS and OS.

Chronic inflammation is known to be associated with tumor development through the induction of oncogenic mutations, genomic instability, early tumor promotion, and enhanced angiogenesis (12). During inflammation, reactive oxygen and nitrogen species are created to combat pathogens, but also damage DNA, which in turn promote mutations that initiate cancer $(13,14)$. Inflammatory responses may act together with oncogenes by driving cells to undergo high levels of transcriptional activity that leads to genome instability (15). Tumorigenesis can be enhanced through increased genomic instability (16). Various cancers induce an inflammatory microenvironment (17). Nutrition is a critical component of immune responses (18). In lung cancer, systemic inflammation, malnutrition, and tumor immune microenvironments are associated with each other, and these are key determinants of tumor progression and treatment response (3). Elevated levels of circulating CRP could be a marker of the increased predisposition to malignancy due to chronic inflammation, a marker of occult cancer leading to inflammation, or both (17).

In the era of immunotherapy in cancer treatment, proper predictive factors of NSCLC patients for immunotherapy have not been developed. The expression of PD-L1 on tumor cells and the TMB have been used in qualifying patients to receive immunotherapy, but not all patients with these predictive factors benefit from immunotherapy (2). Patients with high TMB who received nivolumab and ipilimumab did not show significant survival benefit compared to those who received chemotherapy (19). The presence of immune cells in the anti-tumor immune response such as cluster of differentiation (CD) 8-positive cytotoxic T lymphocytes as 
well as CD4-positive memory and regulatory T lymphocytes has been postulated to be a prognostic marker of the disease course and predictors of activity or modulation of immune system function (20). For simpler prognostic markers, systemic inflammatory marker such as lung immune prognostic index are suggested as biomarker for ICIs in lung cancer. Poor combination of lung immune prognostic index and derived NLR or LDH value are associated with poorer outcomes in patients treated with ICIs (21).

Several studies have demonstrated that systemic inflammatory biomarkers in peripheral blood were predictive markers for treatment outcomes in different solid tumors including prostate, colorectal, and esophageal cancer, melanoma, and NSCLC (22-26). Although the exact biological basis for these findings has not been thoroughly elucidated, inflammatory cells such as neutrophils play a significant role in tumor development and progression via effects on tumor cells or other components of the tumor microenvironment, by secreting chemokines and cytokines such as transforming growth factor- $\beta$, interleukin-6 (IL-6) and matrix metalloproteinases $(27,28)$. CRP is a surrogate marker of IL-6, which is involved in the activation of immune cells, tumor migration and invasion and epithelialto-mesenchymal transition $(29,30)$.

Cancer prognosis is associated with not only tumor staging but also patient-related factors such as nutritional and functional decline. CRP represents systemic inflammation, and albumin reflects both systemic inflammation and the amount of lean tissue $(31,32)$. GPS is a reliable independent prognostic factor in patients with various malignancies and also a marker for predicting prognosis, even in surgery, chemoradiation, and various subgroups incapable of surgery $(5,6)$. In 15 studies including $>2,000$ patients, GPS was associated with increased weight loss, poor performance status, increased comorbidity, increased proinflammatory and angiogenic cytokines, and complications from cancer treatments (5). In resectable lung cancer, the pre- and postoperative GS predicts adverse survival outcomes in patients with resected stage I NSCLC $(33,34)$.

Few previous studies have been conducted on the association between GPS and clinical outcomes in lung cancer patients treated with ICIs. Taichi et al. reported that modified GPS values checked before treatment were associated with shorter OS in NSCLC patients treated with atezolizumab (8). In another study, post-treatment GPS predicted antiprogrammed cell death protein 1 (PD1) treatment (nivolumab or pembrolizumab) efficacy in NSCLC patients (7). Recently, Takamori et al. reported that pretreatment GPS were associated with shorter OS and PFS in NSCLC patients treated with ICIs as first line or higher (9). These studies included populations from single institutions and reported the results of groups treated with anti-PD1 or anti-PD-L1 antibody, or those treated with ICIs as first line therapy. Our study had a moderate sample size and analyzed the prognostic role of pretreatment GPS values in all of the NSCLC patients treated with anti-PD1 or anti-PD-L1 antibodies after failure of platinum-based cytotoxic chemotherapy.

Limitations. This study had some limitations. Firstly, it was a retrospective study. Nonetheless, our study was based on a lung cancer cohort with a moderate sample size using medical records that were faithfully collected from the time of enrollment, and the data were rechecked by authorized data managers. Therefore, data including the baseline characteristics and clinical outcomes were of high quality and reliable. Also, we enrolled lung cancer patients from seven teaching hospitals in the Republic of Korea, so our data represent the Korean general population to some extent. Secondly, since our analyzed results were based on clinical parameters, we could not exactly elucidate the mechanisms of GPS on NSCLC patients treated with immunotherapy. However, hematologic biomarkers are promising predictors of the response to ICIs due to their convenience and accessibility in clinical practice.

\section{Conclusion}

Patients with higher GPS values before treatment showed shorter median irPFS and OS in NSCLC patients treated with ICIs as a second-line or further-line therapy. The pre-treatment serum GPS, along with PD-L1 expression, is a promising value to identify NSCLC patients who could benefit more from ICIs as a second-line or further-line therapy after treatment failure with platinum-based cytotoxic chemotherapy. Further largescale studies are warranted to validate its clinical value.

\section{Conflicts of Interest}

All Authors have completed the ICMJE uniform disclosure form. The Authors have declared that no competing interests exist.

\section{Author's Contributions}

HSK: conception, design, definition of intellectual content, data analysis, statistical analysis, manuscript preparation and manuscript review; CDY: conception, design, definition of intellectual content, data analysis and manuscript review; SHL: conception, design, definition of intellectual content, data analysis and manuscript review; JWK: conception, design, definition of intellectual content, data analysis, manuscript preparation and manuscript review; AYS: literature search, clinical studies, data acquisition and manuscript review; SKK: literature search, clinical studies, data acquisition, manuscript preparation and manuscript review; CKP: literature search, clinical studies, data acquisition and manuscript review; JSK: literature search, clinical studies, data acquisition and manuscript review; SJK: literature search, clinical studies, data acquisition, manuscript preparation and manuscript review. 


\section{Acknowledgements}

The Authors would like to acknowledge and thank Bora Kwon, data manager for help with data collection and management of lung cancer registry.

\section{References}

1 Doroshow DB, Sanmamed MF, Hastings K, Politi K, Rimm DL, Chen L, Melero I, Schalper KA and Herbst RS: Immunotherapy in non-small cell lung cancer: facts and hopes. Clin Cancer Res 25(15): 4592-4602, 2019. PMID: 30824587. DOI: 10.1158/ 1078-0432.CCR-18-1538

2 Wojas-Krawczyk K, Kalinka E, Grenda A, Krawczyk P and Milanowski J: Beyond PD-L1 markers for lung cancer immunotherapy. Int J Mol Sci 20(8): 1915, 2019. PMID: 31003463. DOI: $10.3390 / \mathrm{ijms} 20081915$

3 Alifano M, Mansuet-Lupo A, Lococo F, Roche N, Bobbio A, Canny E, Schussler O, Dermine H, Régnard JF, Burroni B, Goc J, Biton J, Ouakrim H, Cremer I, Dieu-Nosjean MC and Damotte D: Systemic inflammation, nutritional status and tumor immune microenvironment determine outcome of resected nonsmall cell lung cancer. PLoS One 9(9): e106914, 2014. PMID: 25238252. DOI: 10.1371/journal.pone.0106914

4 Hu M, Xu Q, Yang S, Han S, Zhu Y, Lin Q, Sun X, Liu H and $\mathrm{Xu}$ Y: Pretreatment systemic inflammation response index (SIRI) is an independent predictor of survival in unresectable stage III non-small cell lung cancer treated with chemoradiotherapy: a two-center retrospective study. Ann Transl Med 8(20): 1310, 2020. PMID: 33209890. DOI: 10.21037/atm-20-6484

5 McMillan DC: The systemic inflammation-based Glasgow Prognostic Score: a decade of experience in patients with cancer. Cancer Treat Rev 39(5): 534-540, 2013. PMID: 22995477. DOI: $10.1016 /$ j.ctrv.2012.08.003

6 Simmons CPL, McMillan DC, McWilliams K, Sande TA, Fearon KC, Tuck S, Fallon MT and Laird BJ: Prognostic tools in patients with advanced cancer: a systematic review. J Pain Symptom Manage 53(5): 962-970.e10, 2017. PMID: 28062344. DOI: $10.1016 /$ j.jpainsymman.2016.12.330

7 Kasahara N, Sunaga N, Tsukagoshi Y, Miura Y, Sakurai R, Kitahara S, Yokobori T, Kaira K, Mogi A, Maeno T, Asao T and Hisada T: Post-treatment Glasgow Prognostic Score predicts efficacy in advanced non-small-cell lung cancer treated with anti-PD1. Anticancer Res 39(3): 1455-1461, 2019. PMID: 30842182. DOI: 10.21873 /anticanres.13262

8 Matsubara T, Takamori S, Haratake N, Toyozawa R, Miura N, Shimokawa M, Yamaguchi M, Seto T and Takenoyama M: The impact of immune-inflammation-nutritional parameters on the prognosis of non-small cell lung cancer patients treated with atezolizumab. J Thorac Dis 12(4): 1520-1528, 2020. PMID: 32395289. DOI: $10.21037 /$ jtd.2020.02.27

9 Takamori S, Takada K, Shimokawa M, Matsubara T, Fujishita T, Ito K, Toyozawa R, Yamaguchi M, Okamoto T, Yoneshima Y, Tanaka K, Okamoto I, Tagawa T and Mori M: Clinical utility of pretreatment Glasgow prognostic score in non-small-cell lung cancer patients treated with immune checkpoint inhibitors. Lung Cancer 152: 27-33, 2021. PMID: 33341085. DOI: 10.1016/ j.lungcan.2020.11.026

10 McMillan DC, Crozier JE, Canna K, Angerson WJ and McArdle CS: Evaluation of an inflammation-based prognostic score
(GPS) in patients undergoing resection for colon and rectal cancer. Int J Colorectal Dis 22(8): 881-886, 2007. PMID: 17245566. DOI: 10.1007/s00384-006-0259-6

11 Kang HS, Lee HY, Jung JI, Kim JS, Kim YH, Kim SJ, Kim SC, Kwon SS, Kim YK and Kang JY: Clinical significance of Glasgow Prognostic Score in patients with tuberculous pleurisy. J Thorac Dis 10(11): 6077-6087, 2018. PMID: 30622779. DOI: 10.21037/jtd.2018.10.02

12 Arbour KC and Riely GJ: Systemic therapy for locally advanced and metastatic non-small cell lung cancer: a review. JAMA 322(8): 764-774, 2019. PMID: 31454018. DOI: 10.1001/ jama.2019.11058

13 Kay J, Thadhani E, Samson L and Engelward B: Inflammationinduced DNA damage, mutations and cancer. DNA Repair (Amst) 83: 102673, 2019. PMID: 31387777. DOI: 10.1016/ j.dnarep.2019.102673

14 Lin R, Zhang C, Zheng J, Tian D, Lei Z, Chen D, Xu Z and Su M: Chronic inflammation-associated genomic instability paves the way for human esophageal carcinogenesis. Oncotarget 7(17): 24564-24571, 2016. PMID: 27028857. DOI: 10.18632/ oncotarget. 8356

15 Tubbs A and Nussenzweig A: Endogenous DNA damage as a source of genomic instability in cancer. Cell 168(4): 644-656, 2017. PMID: 28187286. DOI: 10.1016/j.cell.2017.01.002

16 Champeris Tsaniras S, Villiou M, Giannou AD, Nikou S, Petropoulos M, Pateras IS, Tserou P, Karousi F, Lalioti ME, Gorgoulis VG, Patmanidi AL, Stathopoulos GT, Bravou V, Lygerou $\mathrm{Z}$ and Taraviras S: Geminin ablation in vivo enhances tumorigenesis through increased genomic instability. J Pathol 246(2): 134-140, 2018. PMID: 29952003. DOI: 10.1002/path.5128

17 Allin KH and Nordestgaard BG: Elevated C-reactive protein in the diagnosis, prognosis, and cause of cancer. Crit Rev Clin Lab Sci 48(4): 155-170, 2011. PMID: 22035340. DOI: 10.3109/ 10408363.2011.599831

18 Chandra RK: Nutrition and the immune system: an introduction. Am J Clin Nutr 66(2): 460S-463S, 1997. PMID: 9250133. DOI: 10.1093/ajen/66.2.460S

19 Remon J, Esteller L and Taus Á: Nivolumab plus ipilimumab combination therapy for the first-line treatment NSCLC: evidence to date. Cancer Manag Res 11: 4893-4904, 2019. PMID: 31213908. DOI: 10.2147/CMAR.S164935

20 Fridman WH, Zitvogel L, Sautès-Fridman C and Kroemer G: The immune contexture in cancer prognosis and treatment. Nat Rev Clin Oncol 14(12): 717-734, 2017. PMID: 28741618. DOI: 10.1038/nrclinonc.2017.101

21 Varga A, Bernard-Tessier A, Auclin E, Mezquita Pérez L, Baldini C, Planchard D, Marabelle A, Hollebecque A, Besse B and Massard C: Applicability of the lung immune prognostic index (LIPI) in patients with metastatic solid tumors when treated with immune checkpoint inhibitors (ICI) in early clinical trials. Annals of Oncology 30: i2, 2020. DOI: 10.1093/annonc/mdz027.001

22 Bilen MA, Martini DJ, Liu Y, Shabto JM, Brown JT, Williams M, Khan AI, Speak A, Lewis C, Collins H, Kissick HT, Carthon BC, Akce M, Shaib WL, Alese OB, Pillai RN, Steuer CE, Wu CS, Lawson DH, Kudchadkar RR, El-Rayes BF, Ramalingam SS, Owonikoko TK, Harvey RD and Master VA: Combined effect of sarcopenia and systemic inflammation on survival in patients with advanced stage cancer treated with immunotherapy. Oncologist 25(3): e528-e535, 2020. PMID: 32162807. DOI: 10.1634/theoncologist.2019-0751 
23 Liang S, Li C, Gao Z, Li J, Zhao H, Yu J and Meng X: A nomogram to predict short-term outcome of radiotherapy or chemoradiotherapy based on pre/post-treatment inflammatory biomarkers and their dynamic changes in esophageal squamous cell carcinoma. Int Immunopharmacol 90: 107178, 2021. PMID: 33218939. DOI: $10.1016 /$ j.intimp.2020.107178

24 Maruyama T, Shimoda M, Hakoda H, Sako A, Ueda K and Suzuki S: Preoperative prognostic nutritional index predicts risk of recurrence after curative resection for stage IIA colon cancer. Am J Surg 222(1): 179-185, 2021. PMID: 33138968. DOI: 10.1016/j.amjsurg.2020.10.032

25 Santos PB, Patel H, Henrique R and Félix A: Can epigenetic and inflammatory biomarkers identify clinically aggressive prostate cancer? World J Clin Oncol 11(2): 43-52, 2020. PMID: 32133274. DOI: $10.5306 /$ wjco.v11.i2.43

26 Xia WY, Zhu XR, Feng W, Liu J, Wang JM, Lv CX, Zhang Q, Yu W, Cai XW and Fu XL: Neutrophil-lymphocyte ratio and platelet-lymphocyte ratio associations with heart and body dose and their effects on patient outcomes in locally advanced nonsmall cell lung cancer treated with definitive radiotherapy. Transl Lung Cancer Res 9(5): 1996-2007, 2020. PMID: 33209619. DOI: $10.21037 /$ tlcr-20-831

27 Treffers LW, Hiemstra IH, Kuijpers TW, van den Berg TK and Matlung HL: Neutrophils in cancer. Immunol Rev 273(1): 312328, 2016. PMID: 27558343. DOI: 10.1111/imr.12444

28 Templeton AJ, McNamara MG, Šeruga B, Vera-Badillo FE, Aneja P, Ocaña A, Leibowitz-Amit R, Sonpavde G, Knox JJ, Tran B, Tannock IF and Amir E: Prognostic role of neutrophilto-lymphocyte ratio in solid tumors: a systematic review and meta-analysis. J Natl Cancer Inst 106(6): dju124, 2014. PMID: 24875653. DOI: 10.1093/jnci/dju124

29 Ozawa Y, Amano Y, Kanata K, Hasegwa H, Matsui T, Kakutani T, Koyauchi T, Tanahashi M, Niwa H, Yokomura K and Suda T: Impact of early inflammatory cytokine elevation after commencement of PD-1 inhibitors to predict efficacy in patients with non-small cell lung cancer. Med Oncol 36(4): 33, 2019. PMID: 30825015. DOI: 10.1007/s12032-019-1255-3
30 Liu W, Wang H, Bai F, Ding L, Huang Y, Lu C, Chen S, Li C, Yue $\mathrm{X}$, Liang $\mathrm{X}$, Ma $\mathrm{C}, \mathrm{Xu} \mathrm{L}$ and Gao L: IL-6 promotes metastasis of non-small-cell lung cancer by up-regulating TIM4 via NF-kB. Cell Prolif 53(3): e12776, 2020. PMID: 32020709. DOI: $10.1111 /$ cpr.12776

31 McMillan DC, Elahi MM, Sattar N, Angerson WJ, Johnstone J and McArdle CS: Measurement of the systemic inflammatory response predicts cancer-specific and non-cancer survival in patients with cancer. Nutr Cancer 41(1-2): 64-69, 2001. PMID: 12094630. DOI: 10.1080/01635581.2001.9680613

32 McMillan DC, Watson WS, O'Gorman P, Preston T, Scott HR and McArdle CS: Albumin concentrations are primarily determined by the body cell mass and the systemic inflammatory response in cancer patients with weight loss. Nutr Cancer 39(2): 210-213, 2001. PMID: 11759282. DOI: 10.1207/S15327914nc392_8

33 Lindenmann J, Fink-Neuboeck N, Taucher V, Pichler M, Posch F, Brcic L, Smolle E, Koter S, Smolle J and Smolle-Juettner FM: Prediction of postoperative clinical outcomes in resected stage I non-small cell lung cancer focusing on the preoperative Glasgow Prognostic Score. Cancers (Basel) 12(1): 152, 2020. PMID: 31936329. DOI: 10.3390/cancers12010152

34 Tomita M, Ayabe T, Chosa E and Nakamura K: Prognostic significance of pre- and postoperative glasgow prognostic score for patients with non-small cell lung cancer. Anticancer Res 34(6): 3137-3140, 2014. PMID: 24922684.
Received July 29, 2021

Revised September 23, 2021

Accepted September 27, 2021 